\title{
THE ASYMPTOTICS OF THE MITTAG-LEFFLER POLYNOMIALS
}

\author{
R. B. PARIS
}

Abstract. We investigate the asymptotic behaviour of the Mittag-Leffler polynomials $G_{n}(z)$ for large $n$ and $z$, where $z$ is a complex variable satisfying $0 \leqslant \arg z \leqslant \frac{1}{2} \pi$. A summary of the asymptotic properties of $G_{n}(i x)$ for real values of $x$ and an approximation for its extreme zeros as $n \rightarrow \infty$ are given. When the variables are such that $z / n$ is finite, an expansion is obtained using the method of steepest descents applied to a suitable integral representation. This expansion holds everywhere in the first quadrant of the $z$-plane except in the neighbourhood of the point $z=i n$, where there is a coalescence of saddle points. Numerical results are presented to illustrate the accuracy of the various expansions.

Mathematics subject classification (2010): Primary 41A60, 33C45.

Keywords and phrases: Mittag-Leffler polynomials, asymptotic expansion, uniform approximation, extreme zeros.

\section{REFERENCES}

[1] H. Bateman, The polynomial of Mittag-Leffler, Proc. N. A. S. 26 (1940), 491-496.

[2] H. Bateman, An orthogonal property of the hypergeometric polynomial, Proc. N. A. S. 28 (1942), 374-377.

[3] N. Bleistein and R. A. Handelsman, Asymptotic Expansion of Integrals, Dover, New York, 1986.

[4] R. B. Dingle, Asymptotic Expansions: Their Derivation and Interpretation, Academic Press, London, 1973

[5] T. X. He, L. C. Hsu And P. J.-S. Shiue, The Scheffer group and the Riordan group, Discrete Appl. Math. 155 (2007), 1895-1909.

[6] X. Li AND R. Wong, On the asymptotics of the Meixner-Pollaczek polynomials and their zeros, Constr. Approx. 17 (2001), 59-90.

[7] Y. L. Luke, The Special Functions and Their Approximations, Vol. 1, Academic Press, New York, 1969.

[8] F. W. J. Olver, Asymptotics and Special Functions, Academic Press, New York, 1974. Reprinted in A. K. Peters, Massachussets, 1997.

[9] F. W. J. Olver, D. W. Lozier, R. F. Boisvert and C. W. Clark (eds.), NiST Handbook of Mathematical Functions, Cambridge University Press, Cambridge, 2010.

[10] R. B. PARIS, Hadamard Expansions and Hyperasymptotic Evaluation: an Extension of the Method of Steepest Descents, Encyclopedia of Mathematics, vol. 141, Cambridge University Press, Cambridge, 2011.

[11] S. Roman, The Umbral Calculus, Academic Press, New York, 1984.

[12] G. RZA̧DKOWSKI, On some expansions, involving falling factorials, for the Euler gamma function and the Riemann zeta function, arXiv.org/abs/1007.1955v1 (2010).

[13] M. S. Stanković, S. D. Marinković and P. M. Rajković, Deformed Mittag-Leffler polynomials, arXiv.org/abs/1007.3612v1 (2010).

[14] G. N. Watson, Asymptotic expansions of hypergeometric functions, Trans. Cambridge Phil. Soc. 22 (1918), 277-308.

[15] E. T. Whittaker And G. N. Watson, Modern Analysis, Cambridge University Press, Cambridge 1952.

[16] R. Wong, Asymptotic Expansion of Integrals, Academic Press, London, 1989. 